\title{
Molecular characterisation of Cryptosporidium (Apicomplexa) in children and cattle in Romania
}

\author{
Patrícia Manuela Vieira $^{1}$, Narcisa Mederle ${ }^{2}$, Maria Luísa Lobo $^{1}$, Kálmán Imre $^{3}$, Ovidiu Mederle ${ }^{4}$, Lihua Xiao $^{5}$, \\ Gheorghe Darabus ${ }^{2}$ and Olga Matos ${ }^{1}$
}

\author{
${ }^{1}$ Unidade de Parasitologia Médica, Grupo de Protozoários Oportunistas/VIH e Outros Protozoários, CMDT, Instituto de Higiene e \\ Medicina Tropical, Universidade Nova de Lisboa, Portugal; \\ ${ }^{2}$ Department of Parasitology and Parasitic Diseases, Faculty of Veterinary Medicine, Faculty of Veterinary Medicine, Banat's \\ University of Agricultural Sciences and Veterinary Medicine Timisoara, Timisoara, Romania; \\ ${ }^{3}$ Department of Food Hygiene and Veterinary Public Health, Faculty of Veterinary Medicine, Banat's University of Agricultural \\ Sciences and Veterinary Medicine, Timisoara, Romania; \\ ${ }^{4}$ Faculty of Medicine, University of Medicine and Pharmacy, Timisoara, Romania; \\ ${ }^{5}$ Division of Foodborne, Waterborne and Environmental Diseases, Centers for Disease Control and Prevention, Atlanta, GA, USA
}

\begin{abstract}
To investigate the transmission of species of Cryptosporidium Tyzzer, 1907 in Timis County, Romania, 48 isolates of Cryptosporidium coccidia from 11 children, 29 calves and eight pigs were characterised by molecular analysis of two loci (SSU rRNA and 60-kDa glycoprotein gene). Overall, 22 isolates were amplified and sequence analyses revealed that all isolates were Cryptosporidium parvum Tyzzer, 1912. Two subtype families were identified, IIa and IId. Subtype IIdA22G1 ( $\mathrm{n}=4)$ was the single $C$. parvum subtype found in children. Subtypes found in calves included IIdA27G1 $(\mathrm{n}=8)$, a novel subtype, IIdA25G1 $(\mathrm{n}=5)$, IIdA22G1 ( $=2)$, IIdA21G1a $(n=1)$, and IIaA16G1R1 $(n=1)$. Subtype IIdA26G1 was found in a pig. These results were significantly different from previous Romanian reports, as the five subtypes of family IId identified in this study were never identified previously in this country. Thus, cattle may be a source of Cryptosporidium infections for humans and the transmission dynamics of C. parvum in Romania is more complex than previously believed.
\end{abstract}

Keywords: Cryptosporidium parvum, man, calves, molecular epidemiology, GP60 variability, eastern Europe

Protists of the genus Cryptosporidium Tyzzer, 1907 are important etiological agents of gastrointestinal disease of a wide range of vertebrate animals, including humans (Xiao et al. 2004, Fayer 2010). Cryptosporidium infection may be acquired by direct contact with infected persons or animals, and indirectly by ingestion of contaminated water and food (Fayer 2010, Xiao 2010). Cryptosporidium includes over 20 species (Xiao et al. 2004, Fayer 2010), with Cryptosporidium hominis Morgan-Ryan, Fall, Ward, Hijjawi, Sulaiman et al., 2002, which has anthroponotic transmission and Cryptosporidium parvum Tyzzer, 1912 with zoonotic or anthroponotic transmission as the two most common species infecting humans (Xiao 2010). Cryptosporidium parvum, Cryptosporidium bovis Fayer, Santín et Xiao, 2005, Cryptosporidium ryanae Fayer, Santín et Trout, 2008 and Cryptosporidium andersoni Lindsay, Upton, Owens, Morgan, Mead et al., 2000 were described from cattle, with an age-related distribution (Santín et al. 2004, Fayer et al. 2007). Cryptosporidium parvum is the major zoonotic species and is the dominant one in pre-weaned calves until two months of age (Santín et al. 2004). Natural infections in pigs by Cryptosporidium spp. have also been reported and are commonly attributable to two species (Xiao 2010), Cryptosporidium suis Ryan, Monis, Enemark, Sulaiman, Samarasinghe et al., 2004 and Cryptosporidium scrofarum Kváč, Kestránová, Pinková, Květoňová, Kalinová et al., 2013 (Fayer 2010, Kváč et al. 2013).

The present study was undertaken to genetically characterise Cryptosporidium spp. from humans and animals (Timis County, Romania), map the diversity of Cryptosporidium species in both populations and identify potential source(s) of human infection.

In this study we used 48 faecal samples from 48 individuals diagnosed as positive for Cryptosporidium spp. by a modified Ziehl-Neelsen staining. All faecal samples were collected in Timis County, Romania (2008), including 11 specimens from children under 12 years of age with diarrhea, 29 specimens from calves up to two months old and eight from nine-week-old pigs.

Address for correspondence: O. Matos, Instituto de Higiene e Medicina Tropical, Rua da Junqueira n. ${ }^{\circ} 100,1349-008$ Lisboa, Portugal. Phone: +351 2136526 00; Fax: +351 2136321 05; E-mail: omatos@ihmt.unl.pt 


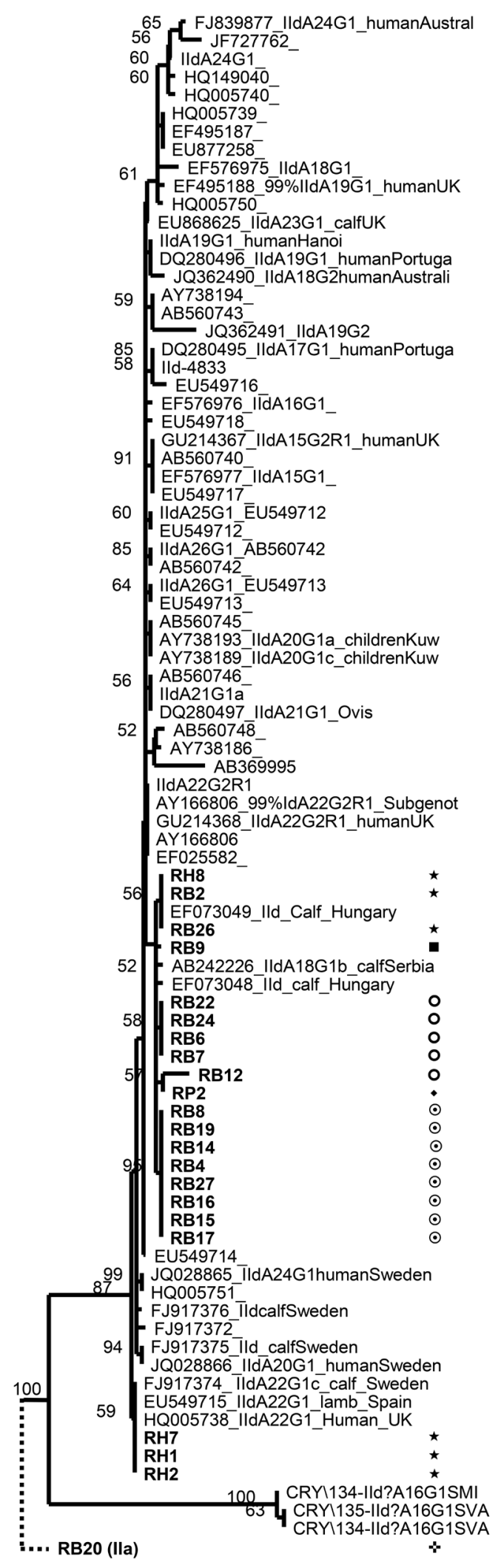

Fig. 1. Phylogenetic relationship between Cryptosporidium parvum subtypes characterised in this study and other sequences in GenBank, inferred by a neighbour-joining analysis of GP60 sequences, based on genetic distance calculated by Kimura twoparameter model. Numbers above branches indicate the confidence values obtained by bootstrap analysis with 1000 replicates. Abbreviations: RB - bovine faecal sample; $\mathrm{RH}$ - human faecal sample; RP - pig faecal sample; $\star$ - IIdA22G1; - IIdA21G1a; O - IIdA25G1; • - IIdA26G1, ๑ - IIdA27G1, † - IIaA16G1R1.
Stool samples from the animals were collected directly from the rectum. Samples were stored in $2.5 \%$ potassium dichromate at $4{ }^{\circ} \mathrm{C}$. The oocysts from samples were concentrated by a modified water-ether sedimentation method. The DNA was extracted by a MiniBeadBeater/sílica method (Alves et al. 2001, 2003).

The identification of Cryptosporidium species and genotypes was performed by nested-PCR analysis of an $830 \mathrm{bp}$ fragment of the SSU rRNA gene (Xiao and Ryan 2008). Subtyping analysis was done by nested-PCR analysis of a $400 \mathrm{bp}$ fragment of the GP60 gene (Alves et al. 2003). The secondary PCR products were separated on $1.5 \%$ agarose gel and visualised by ethidium bromide staining under UV light.

All secondary SSU rRNA and GP60 products were purified with Jetquick Gel Extraction Spin Kit ${ }^{\circledR}$ (Genomed, Löhne, Germany) and sequenced in both directions on an ABI 3730XL automated sequencer (Applied Biosystems, Foster City, CA, USA). The sequences obtained in this study were aligned with reference sequences of Cryptosporidium spp. from the GenBank database using the MultAlin (http://multalin.toulouse.inra.fr/multalin/) software for species/genotypes and subtype identification. The $C$. parvum subtypes were named according to the number of TCA and TCG repeats in the trinucleotide repeat region and the mutations in the nonrepeating regions (Xiao 2010). To evaluate phylogenetic relationship among various $C$. parvum subtypes characterised in this and other studies, a neighbour-joining tree was constructed from the aligned sequences using the Treecom W program, based on genetic distance calculated by Kimura twoparameter model. The reability of branches was assessed by bootstrap analysis with 1000 replicates. The partial GP60 sequences generated in this study were deposited in the GenBank database (Acc. Nos. KF500407 and KC469687-KC469694).

Amplification of Cryptosporidium DNA was successfully achieved for 14 (two specimens from children and 12 from calves) of the 48 specimens diagnosed as positive for Cryptosporidium spp. by microscopy. The subsequent sequencing and alignment of all our nucleotide sequences with others of known identity deposited in GenBank database showed that all sequenced isolates belonged to C. parvum. Of the 14 isolates, six had $100 \%$ homology with the C. parvum nucleotide sequence DQ656355, six had $100 \%$ similarity with the $C$. parvum nucleotide sequence AB513881 and two had $100 \%$ homology with the $C$. parvum nucleotide sequence JN247404.

PCR products of the GP60 gene were obtained for 22 (four specimens from children, 17 from calves and one from a pig) of the 48 C. parvum isolates. The alignment of the sequences with reference sequences from GenBank confirmed that C. parvum was the only species found in the samples. The GP60 sequences of the 22 isolates confirmed six subtypes belonging to two zoonotic subtype families, IIa and IId. Four of the IId subtypes, IIdA21G1a, IIdA22G1, IIdA25G1 and IIdA26G1, are known subtypes, with GenBank Acc. Nos. AB560746, EU549715, EU549712 and EU549713. A fifth subtype, IIdA27G1, was a novel subtype identified in this study. The IIa subtype characterised in this study (IIaA16G1R1) had 100\% homology to sequence AY149615 deposited in the GenBank database.

The remaining sequences (subtypes of subtype family IId) had only $99 \%$ homology to the sequences in GenBank with at least one polymorphism in the non-repeating region. The sequences from RB4, RB8, RB14, RB15, RB16, RB17, RB19 and RB27, 
Table 1. Distribution of Cryptosporidium parvum GP60 subtypes in humans and animals in Timis County, Romania.

\begin{tabular}{lccc}
\hline \multirow{2}{*}{ Subtypes } & \multicolumn{3}{c}{ Host } \\
\cline { 2 - 4 } & $\begin{array}{c}\text { Man } \\
(n=4)\end{array}$ & $\begin{array}{c}\text { Calf } \\
(n=17)\end{array}$ & $\begin{array}{c}\text { Pig } \\
(n=1)\end{array}$ \\
\hline IIaA16G1R1 & 0 & 1 & 0 \\
IIdA21G1a & 0 & 1 & 0 \\
IIdA22G1 & 4 & 2 & 0 \\
IIdA25G1 & 0 & 5 & 0 \\
IIdA26G1 & 0 & 0 & 1 \\
IIdA27G1 & 0 & 8 & 0 \\
\hline
\end{tabular}

all identified as the new subtype IIdA27G1, exhibited intra subtype sequence polymorphism. According to the nomenclature proposed by Sulaiman et al. (2005) and Xiao (2010), we assigned these new subtypes as IIdA21G1b (RB9), IIdA22G1b (RH1, RH2, RH7), IIdA22G1c (RH8, RB2, RB26), IIdA25G1b (RB6, RB7), IIdA25G1c (RB22), IIdA26G1b (RP2), IIdA27G1a (RB4, RB14, RB16, RB19) and IIdA27G1b (RB8, RB15, RB27). Moreover, the multiple alignment of GP60 sequences obtained shows that although all sequences of subtype family IId are identical to each other in the non-repeating region, sequences of seven isolates stand out from others due to the existence of at least one polymorphism in the non-repeating region. Phylogenetic analysis of the sequences confirmed that $C$. parvum isolates belonged to two subtype families (IIa and IId) and showed the existence of two genetically distinct types of sequences in the subtype IIdA22G1 (Fig. 1). One of them was described in three human isolates (RH1, RH2 and RH7), whereas the second type of sequence was detected in one human (RH8) and two bovine isolates (RB26 and RB2).

Subtype family IId was prevalent and widely distributed in the present study. Of the 22 isolates, 96\% (21/22) belonged to this subtype family, being detected in humans (4 isolates), calves (16) and pigs (1). In contrast, subtype family IIa was less prevalent: of the 22 isolates, only one was detected in a calf. As shown in Table 1, IIdA22G1 was the only subtype detected in humans (4 isolates). In contrast, multiple subtypes were seen in calves: IIdA27G1 was the predominant ( 8 isolates from a total of 17 samples), followed by subtypes IIdA25G1 (5 isolates) and IIdA22G1 (2), whereas subtypes IIaA16G1R1 and IIdA21G1a were rare (only 1 isolate). The only specimen characterised in pigs had the subtype IIdA26G1.

Genetic characterisation of Cryptosporidium isolates from Timis County, Romania, revealed that $C$. parvum was the only species present in children. Our results are in agreement with several studies conducted in European countries (France, Portugal, Slovenia, Switzerland, UK), which have shown a common occurrence of C. parvum in humans (McLauchlin et al. 2000, Morgan et al. 2000; Alves et al. 2001, 2003, Guyot et al. 2001, Matos et al. 2004, Soba and Logar 2008). In contrast, C. hominis is more prevalent in other regions of the world, especially in developing countries, Australia and USA (Xiao 2010).

In calves, all isolates were characterised as C. parvum, as in previous studies conducted in the same region (Imre et al. 2011). Our results correlate with those on genotyping of Cryptosporidium spp. in calves of the same age, in which C. parvum was considered responsible for most infections (Xiao 2010). The high prevalence of C. parvum detected in this study is consistent with the age of the calves, which may serve as major reservoirs of the most important zoonotic species, C. parvum.

In pigs, a single isolate was identified as $C$. parvum, whereas the species most often detected in pigs are C. suis and C. scrofarum (see Kváč et al. 2013). Cryptosporidium parvum has been only detected in a few studies (Kváč et al. 2009, Xiao 2010, Garcia-Presedo et al. 2013, Němejc et al. 2013).

The present study reveals the existence of some genetic diversity in C. parvum in Romania. Overall, isolates of C. parvum were from two subtype families, IIa and IId, and six subtypes, namely IIaA16G1R1, IIdA21G1a, IIdA22G1, IIdA25G1, IIdA26G1 and IIdA27G1. All subtypes were only seen in calves, except subtype IIdA22G1 in both calves and humans, and subtype IIdA26G1, in a pig. Interestingly, the subtype family IIa was only seen in a single isolate of C. parvum, being identified as the subtype IIaA16G1R1. This subtype, which is an important etiological agent for bovine cryptosporidiosis (Xiao 2010), is predominant in countries neighbouring Romania (71\% in Hungary, but only 21 samples examined; 33\% in Serbia and Montenegro, 18 samples; $13 \%$ in Slovenia, 6 samples) (Plutzer and Karanis 2007, Misic and Abe 2007, Soba and Logar 2008), suggesting that in these countries as well as in Romania, this subtype is an important etiological agent for bovine cryptosporidiosis (Plutzer and Karanis 2007, Soba and Logar 2008, Imre et al. 2011). Besides calves, this subtype has also been found in lambs, pigs and humans and thus can be considered as a zoonotic pathogen (Soba and Logar 2008, Kváč et al. 2009, Imre et al. 2013). In our study, the subtype IIaA15G2R1, described as the most widely distributed in calves worldwide (Xiao 2010), was not identified.

Amongst the C. parvum IId subtype families characterised, five different subtypes were detected for the first time in Romania. Interestingly, some of these subtypes, namely IIdA21G1a, IIdA25G1 and IIdA26G1, have been found only occasionally in small ruminants (Xiao 2010), except for subtype IIdA22G1, which has also occasionally been implicated in bovine cryptosporidiosis in some European countries (Belgium, Germany, Hungary, Sweden - Silverlås et al. 2010, 2012, Xiao 2010). Apart from calves, this subtype was also detected in children with gastrointestinal symptoms in the present study. Subtype IIdA22G1 was also previously seen in humans in Portugal and in the United Kingdom (Alves et al. 2006, Chalmers et al. 2011). Results of phylogenetic analysis of the GP60 gene revealed the existence of two types of sequence within this subtype. One of these types from three human isolates (RH1, RH2 and RH7) was related to, but not identical with, isolates from lambs in Spain (GenBank Acc. No EU549715) and bovines in Sweden (GenBank Acc. No. FJ917374). The fact that this type of sequence was found only in humans in the present study indicates the possibility of anthroponotic transmission. In contrast, the second type of sequences was seen in a human (RH8) and two bovine isolates (RB26 and RB2). It should be noted that subtypes IIdA21G1a and IIdA26G1 were also already found in human isolates (Alves et al. 2006, Nazemalhosseini-Mojarad et al. 2011). The subtype IIdA27G1 was the most frequent subtype in calves in this study, and represents a new subtype.

In summary, our epidemiological analysis suggests that in Timis County, Romania, there is a high prevalence of C. parvum subtype family IId characterised in this study, in contrast to 
the high prevalence of the family IIa in previous studies also in this region (Imre et al. 2011). Human cryptosporidiosis appears to occur primarily through zoonotic transmission and cattle appear to be a major zoonotic reservoir. Results of this and previous studies, however, need to be confirmed by analyses of more samples. These studies should also be performed in other regions of Romania and should include better collection of epidemiological data, with the purpose of more conclusive identification of infection sources, reservoir hosts and transmission dynamics of Cryptosporidium spp. in humans in this country.

\section{REFERENCES}

Alves M., Matos O., Pereira da Fonseca I., Delgado E., Lourenço A.M., Antunes F. 2001: Multilocus genotyping of Cryptosporidium isolates from human HIV-infected and animal hosts. J. Eukaryot. Microbiol. Suppl: 17S-18S.

Alves M., Xiao L., Antunes F., Matos O. 2006: Distribution of Cryptosporidium subtypes in humans and domestic and wild ruminants in Portugal. Parasitol. Res. 99: 287-292.

Alves M., Xiao L., Sulaiman I., Lal A.A., Matos O., AnTUNES F. 2003: Subgenotype analysis of Cryptosporidium isolates from humans, cattle, and zoo ruminants in Portugal. J. Clin. Microbiol. 41: 2744-2747.

Chalmers R.M., Smith R.P., Hadfield S.J., Elwin K., Giles M. 2011: Zoonotic linkage and variation in Cryptosporidium parvum from patients in the United Kingdom. Parasitol. Res. 108: $1321-1325$.

FAYER R. 2010: Taxonomy and species delimitation in Cryptosporidium. Exp. Parasitol. 124: 90-97.

Fayer R., Santín M., Trout J.M. 2007: Prevalence of Cryptosporidium species and genotypes in mature dairy cattle on farms in eastern United States compared with younger cattle from the same locations. Vet. Parasitol. 145: 260-266.

García-Presedo I., Pedraza-Díaz S., González-Warleta M., Mezo M., Gómez-Bautista M., Ortega-Mora L.M., Castro-Hermida J.A. 2013: Presence of Cryptosporidium scrofarum, $C$. suis and C. parvum subtypes IIaA16G2R1 and IIaA13G1R1 in Eurasian wild boars (Sus scrofa). Vet. Parasitol. 196: 497-502.

Guyot K., Follet-Dumoulin A., Lelièvre E., Sarfati C., Rabodonirina M., Nevez G., Cailliez J.C., Camus D., DEI-CAS E. 2001: Molecular characterization of Cryptosporidium isolates obtained from humans in France. J. Clin. Microbiol. 39: $3472-3480$.

Imre K., Lobo M.L., Matos O., Popescu C., Genchi C., DĂRĂBUŞ G. 2011: Molecular characterization of Cryptosporidium isolates from pre-weaned calves in Romania: is there an actual risk of zoonotic infections? Vet. Parasitol. 181: 321-324.

Imre K., Luca C., Costache M., Sala C., Morar A., Morariu S., Ilie M.S., Imre M., DĂRĂBuŞ G. 2013: Zoonotic Cryptosporidium parvum in Romanian newborn lambs (Ovis aries). Vet. Parasitol. 191: 119-122.

Kváč M., Kestránová M., Pinková M., Květoňová D., KaLinová J., Wagnerová P., KotKová M., Vítovec J., Ditrich O., McEvoy J., Stenger B., Sak B. 2013: Cryptosporidium scrofarum n. sp. (Apicomplexa: Cryptosporidiidae) in domestic pigs (Sus scrofa). Vet. Parasitol. 191: 218-227.

Kváč M., SaK B., Hanzlíková D., Kotilová J., KvěToŇová D. 2009: Molecular characterization of Cryptosporidium isolates from pigs at slaughterhouses in South Bohemia, Czech Republic. Parasitol. Res. 104: 425-428.

Matos O., Alves M., Xiao L., Cama V., Antunes F. 2004: Cryptosporidium felis and C. meleagridis in persons with HIV, Portugal. Emerg. Infect. Dis. 10: 2256-2257.

Mclauchlin, J., Amar C., Pedraza-Díaz P., Nichols G.L. 2000: Molecular epidemiological analysis of Cryptosporidium spp. in the United Kingdom: results of genotyping Cryptosporidium spp. in 1,705 fecal samples from humans and 105 fecal samples from livestock animals. J. Clin. Microbiol. 38: 3984-3990.

Misic Z., Abe N. 2007: Subtype analysis of Cryptosporidium parvum isolates from calves on farms around Belgrade, Serbia and Montenegro, using the $60 \mathrm{kDa}$ glycoprotein gene sequences. Parasitology 134: 351-358.

Morgan U., Weber R., Xiao L., Sulaiman I., Thompson R.C., Ndiritu W., Lal A., Moore A., Deplazes P. 2000: Molecular characterization of Cryptosporidium isolates obtained from human immunodeficiency virus-infected individuals living in Switzerland, Kenya, and the United States. J. Clin. Microbiol. 38: $1180-1183$.

Nazemalhosseini-Mojarad E., Haghighi A., Taghipour N., Keshavarz A., Mohebi S.R., Zali M.R., Xiao L. 2011: Subtype analysis of Cryptosporidium parvum and Cryptosporidium hominis isolates from humans and cattle in Iran. Vet. Parasitol. 179: 250-252.

NěmejC K., SAK B., Květoňová D., Kernerová N., Rost M., Cama V.A., KváČ M. 2013: Occurrence of Cryptosporidium suis and Cryptosporidium scrofarum on commercial swine farms in the Czech Republic and its associations with age and husbandry practices. Parasitol. Res. 112: 1143-1154.

Plutzer J., Karanis P. 2007: Genotype and subtype analyses of Cryptosporidium isolates from cattle in Hungary. Vet. Parasitol. 146: $357-362$.

Santín M., Trout J.M., Xiao L., Zhou L., Greiner E., Fayer R. 2004: Prevalence and age-related variation of Cryptosporidium species and genotypes in dairy calves. Vet. Parasitol. 122: 103-117.

Sulaiman I.M., Hira P.R., Zhou L., Al-Ali F.M., Al-Shelahi F.A., Shweiki H.M., Iqbal J., Khalid N., Xiao L. 2005: Unique endemicity of cryptosporidiosis in children in Kuwait. J. Clin. Microbiol. 43: 2805-2809.

Silverlås C., Bosaeus-Reineck H., Näslund K., Buörkman C. 2012: Is there a need for improved Cryptosporidium diagnostics in Swedish calves? Int. J. Parasitol. 43: 155-161.

Silverlås C., Näslund K., Buörkman C., Mattsson J.G. 2010: Molecular characterization of Cryptosporidium isolates from Swedish dairy cattle in relation to age, diarrhea and region. Vet. Parasitol. 169: 289-295.

Soba B., Logar J. 2008: Genetic classification of Cryptosporidium isolates from humans and calves in Slovenia. Parasitology 135: $1263-1270$.

XIAO L. 2010: Molecular epidemiology of cryptosporidiosis: an update. Exp. Parasitol. 124: 80-89.

Xiao L., Fayer R., Ryan U., Upton S.J. 2004: Cryptosporidium taxonomy: recent advances and implications for public health. Clin. Microbiol. Rev. 17: 72-97.

XIAO L., Ryan U.M. 2008: Molecular epidemiology. In: R. Fayer and L. Xiao (Eds.), Cryptosporidium and Cryptosporidiosis, Second Edition. CRC Press, Boca Raton, Florida, pp. 119-172. 